\title{
Quasi-Perspectivities in Stable Planes
}

\author{
By
}

\author{
Markus Stroppel, Darmstadt
}

(Received 25 October 1991)

\begin{abstract}
Stable planes are a generalization of compact connected projective planes. The possible configurations of fixed points for quasi-perspectivities are determined (extending results of $\mathrm{R}$. Baer), and restrictions to the structure of finite quasiperspective groups as well as bounds for the dimension of quasi-perspective groups are derived.
\end{abstract}

A stable plane $\mathbb{M}=(M, \mathscr{M})$ is a topological linear incidence geometry such that

- the point space $M$ and the line space $\mathscr{M}$ are locally compact Hausdorff spaces

- the covering dimension $\operatorname{dim} M$ is positive and finite

- the operations $\wedge \mathscr{D} \rightarrow M:(X, Y) \mapsto X \wedge Y$ (intersection of lines) and $\vee: M \times M \backslash\{(x, x) \mid x \in M\} \rightarrow \mathscr{M}:(x, y) \mapsto x y$ (joining of points) are continuous, and the domain $\mathscr{D}$ of definition of $\wedge$ is open in $\mathscr{M} \times \mathscr{M}$.

Familiar examples of stable planes are the geometries induced on open sets of points of compact connected projective planes of finite covering dimension (e.g. the Klein model of the hyperbolic plane, see [5]). There are, however, stable planes that are not embeddable in this way, see [18].

According to a deep result of LöwEN [8], the covering dimension of $M$ equals $2 l$, where $l$ denotes the dimension of a line, and $l$ is one of the integers $1,2,4$, or 8 (i.e. the dimension of one of the real division algebras $\mathbb{R}, \mathbb{C}, \mathbb{H}$, or $\mathbb{D}$ ). The group Aut $(\mathbb{M})$ of all continuous collineations, endowed with the compact-open topology, is a separable, locally compact transformation group on $M$ and $\mathscr{M}$ (see [6], 2.9). This group has proved to be a good tool for the study of stable planes of low dimension (see e.g. [3], [9] and the references given there) and for the 
theory of compact connected projective planes in general (see [14] and the references given there). In order to exploit these techniques for stable planes of arbitrary dimension, one needs information about planar groups (cf. [19]) as well as about quasi-perspectivities.

1. Definitions. a) A subgroup $\Delta \neq 1$ of $\operatorname{Aut}(\mathbb{M})$ is called quasiperspective if each orbit $x^{\Delta}$ is contained in some line $L_{x}$.

b) An element $\delta$ of Aut $(\mathbb{M}$ ) is called quasi-perspective (or a quasiperspectivity) if the subgroup $\langle\delta\rangle$ generated by $\delta$ is quasi-perspective.

c) An element $\delta$ of Aut (M) is called a Baer collineation, if the set Fix $(\delta)$ of fixed points contains a quadrangle and $\operatorname{dim} \operatorname{Fix}(\delta)=$ $=\operatorname{dim} M / 2$.

Note that, for each quasi-perspective group $\Delta$, the line $L_{x}$ is determined uniquely if $x$ is moved by $\Delta$. We write $\mathscr{L}_{\Delta}=$ $=\left\{L_{x} \mid x \in M \backslash\right.$ Fix $\left.(\Delta)\right\}$ and $\mathscr{L}_{\delta}=\mathscr{L}_{\langle\delta\rangle}$ for each quasiperspectivity $\delta$. According to ([7], 1.1), the mapping $\lambda: x \mapsto L_{x}: M \backslash$ Fix $(\Delta) \rightarrow \mathscr{L}_{\Delta}$ is continuous. Restricting $\lambda$ to a line $H$ that is moved by $\Delta$, one obtains that $\mathscr{L}_{\Delta}$ is locally homeomorphic with $H$. Note that each line $L_{x} \in \mathscr{L}_{\Delta}$ is fixed by $\Delta$.

2. Definitions. Let $\Delta$ be a subgroup of $\operatorname{Aut}(\mathbb{M})$, where $\mathbb{M}=(M, \mathscr{M})$ is a stable plane.

a) If $\Delta$ acts trivially on the pencil $\mathscr{M}_{z}=\{L \in \mathscr{M} \mid z \in L\}$ for some point $z \in M$, then $z$ is called the center of $\Delta$.

b) If $\Delta$ acts trivially on some open nonvoid subset $U$ of a line $L_{U}$ in $\mathscr{M}$, then $U$ is called a semi-axis of $\Delta$. If $U=L_{U}$, then $L_{U}$ is called an axis of $\Delta$.

Note that each group $\Delta$ with semi-axis $U$ acts effectively as a group with axis on the open subplane induced on $M \backslash\left(L_{U} \backslash U\right)$.

According to a well-known theorem of R. BAER [1] (see also [11], pp. $71-73$ ), each quasiperspectivity $\delta$ of a projective plane either has center and axis, or the geometry induced on $\operatorname{Fix}(\delta)$ is a non-degenerate projective plane (in fact, a so-called Baer subplane, cf. [4], pp. 82, $91-94)$. In the case of compact connected projective planes of dimension $0<2 l<\infty$, such a subplane has dimension $l$ (see [12], 1.4, [13], 1.4). Note that this follows readily from Löwen's restriction of the possible dimensions and the fact that the set of fixed lines is locally homeomorphic with a line.

We are going to extend Baer's theorem to the case of stable planes. A special feature of stable planes is the following: Removing a proper closed subset $X$ of $M$ (where $(M, \mathscr{M})$ is a stable plane), one obtains a 
stable plane $(M \backslash X, \tilde{M})$, where $\tilde{M}$ consists of those lines of $\mathscr{M}$ that meet $M$ in at least two points. If $\delta$ is an automorphism of $(M, \mathscr{M})$ that leaves $X$ invariant, then $\delta$ induces an automorphism of $(M \backslash X, \tilde{M})$. Therefore one can only expect that $\mathrm{Fix}(\delta)$ forms a part of one of the configurations that occur in projective planes. The following lemma is the key to the characterization of the possible configurations:

3. Lemma. Let $\delta$ be a quasi-perspective automorphism of a stable plane $\mathbb{M}=(M, \mathscr{M})$, and assume that two lines $L_{x}, L_{y} \in \mathscr{L}_{\delta}$ intersect in a point $z \in M$. If $\delta$ is not a Baer collineation, then $z$ is the center of $\delta$.

Proof. We use the continuous mapping $\lambda: M \backslash \operatorname{Fix}(\delta) \rightarrow \mathscr{L}_{\delta}: q \mapsto L_{q}$. Set $l=\operatorname{dim} L_{x}$. In lines $G$ and $H$ through $x$ and $y$, respectively, one finds compact neighbourhoods $X$ of $x$ and $Y$ of $y$ such that $X^{\lambda} \times Y^{\lambda}$ is contained in the domain of definition of $\wedge$. Define the mappings

$$
\begin{aligned}
& \mu: X \rightarrow L_{y}: q \mapsto L_{q} \wedge L_{y}, \\
& v: Y \rightarrow L_{x}: q \mapsto L_{q} \wedge L_{x} .
\end{aligned}
$$

There are two cases: i) There is a point $r \in X^{\mu}$ such that $\operatorname{dim} r^{\mu^{-\lambda}}>l / 2$. Assume that $K \in r^{\mu^{-\lambda}}$ meets $L \in Y^{\lambda}$ in a point $s \neq r$. Then $\operatorname{dim}\left\{L \wedge K^{\prime} \mid K^{\prime} \in r^{\mu^{-} \lambda}\right\}=\operatorname{dim} r^{\mu^{-} \lambda}>l / 2$. Choosing $L^{\prime} \in Y^{\lambda}$ near $L$, one obtains that $\operatorname{Fix}(\delta)$ generates a subplane of dimension greater than $l$. Consequently, $\operatorname{dim} \operatorname{Fix}(\delta)=\operatorname{dim} M$, and $\operatorname{Fix}(\delta)$ is a neighbourhood in $M$ by ([8], Theorem 11c)). Now $\delta=1$, a contradiction. Therefore $Y^{v}=\{z\}=X^{\mu}$, and $r=z$. Since $L_{x}$ was arbitrary, we obtain that $\mathscr{L}_{\delta} \cap \mathscr{M}_{z}$ is open in $\mathscr{M}_{z}$. On the other hand, $\mathscr{L}_{\delta}$ is closed in $\mathscr{M}$. Now $\mathscr{L}_{\delta} \cap \mathscr{M}_{z}=\mathscr{M}_{z}$ since $\mathscr{M}_{z}$ is connected ([6], 1.14), and $z$ is the center of $\delta$.

ii) In the remaining case we have that $\max \left\{\operatorname{dim} r^{\mu^{-}} \mid r \in X^{\mu}\right\} \leqslant l / 2$. An application of the dimension formula in ([10], III.6) yields that $\operatorname{dim} X^{\mu} \geqslant l / 2$, and the set $\left\{K \wedge L \mid(K, L) \in X^{\mu} \times Y^{\nu}\right\}$ generates a subplane of dimension $d \geqslant l$. Now $d=l$ since $\delta \neq 1$, and $\delta$ is a Baer collineation.

We wish to extend R. Baer's theorem to the case of quasi-perspectivities of prime order, in particular involutions. For this purpose, we shall use the following topological lemma.

4. Lemma. Let $p \in M$, where $(M, \mathscr{M})$ is a stable plane. Then $\mathscr{M}_{p}\{L\}$ has trivial homology groups for each line L through $p$. 
Proof. According to ([8], 5.3) each compact subset $\mathscr{U}$ of $\mathscr{M}_{p} \backslash\{L\}$ has a contractible compact neighbourhood in $\mathscr{M}_{p}\{\{L$. Hence all homotopy groups are trivial, and the assertion follows from Hurwitz' Theorem ([17], Theorem 4, p. 397).

5. Theorem. Let $\delta \neq 1$ be a quasi-perspectivity (e.g. an involution). Then one (and only one) of the following holds:

a) $\delta$ is free, i.e. $\operatorname{Fix}(\delta)=\emptyset$.

b) $\delta$ is a Baer collineation.

c) $\delta$ has a center or an axis (or both).

d) $\operatorname{Fix}(\delta)$ is contained in a unique line $L$, which is not an axis; and through each fixed point there passes no other fixed line except $L$.

If $\delta$ has prime order (in particular if $\delta$ is an involution), case d) does not occur.

Proof. The cases a), b), c) and d) are mutually exclusive. Let $q \in \operatorname{Fix}(\delta)$. Since Fix $(\delta)$ is not open in $M$, there is a sequence $q_{n}$ converging to $q$ such that $q_{n} \notin$ Fix $(\delta)$. According to $([6], 1.17)$ the lines $L_{q_{n}}$ accumulate at a line $L$. This line passes through $q$ by ([6], 1.5). If there is another fixed line $K$ through $q$, then either $K$ or $L$ is an axis, or 3) applies. Since $\mathscr{M}_{q} \backslash\{\mathrm{L}\}$ has trivial homology groups, a result of P. SMITH [15] assures the existence of $K$ in the case where $\delta$ has prime order.

For stable planes, we do not know whether case d) occurs. It is, however, easy to give examples for discrete linear spaces admitting such quasi-perspectivities:

6. Example. Let $(P, \mathscr{P})$ be a projective plane admitting an elation $\tau$ with axis $A \in \mathscr{P}$ and center $c \in A$. For each point $x \in A \backslash\{c\}$, the restriction of $\tau$ to the geometry induced on $P \backslash(A \backslash\{x\})$ is a quasiperspectivity of type d). Note that in the non-discrete case the deleted set is not closed, and we do not obtain a stable plane.

We turn to the study of commuting quasi-perspectivities now.

7. Lemma. Let $\alpha \neq 1$ be a quasi-perspectivity of prime order with axis $A$. Then there is exactly one fixed line $C_{a} \in \mathscr{M}_{a} \backslash\{A\}$ for each point $a \in A$. Moreover, $C_{a}$ belongs to $\mathscr{L}_{\alpha}$.

Proof. The existence of $C_{a}$ follows from [15] since $\mathscr{M}_{a} \backslash\{A\}$ has trivial homology groups (4)). Now $C_{a} \in \mathscr{L}_{\alpha}$, because $C_{a}$ is different from the axis $A$. If there is another fixed line $L \in \mathscr{M}_{a} \backslash\left\{A, C_{a}\right\}$, then $a$ is the center of $\alpha$ by 3). In this case, $C_{b}$ would be an axis for each $b \in A \backslash\{a\}$. 
8. Proposition. Let $\Delta$ be an elementary abelian group of automorphisms of a stable plane $(M, \mathscr{M})$ and assume that there is a line $A \in \mathscr{M}$ such that $A$ is the axis of each $\delta \in \Delta \backslash \mathbf{1}$. Then $\Delta$ is cyclic.

Proof. Choose $\delta \in \Lambda \backslash \mathbf{1}$, and let $a$ be a point on $A$. By 7), there are exactly two lines $A$ and $C_{a}$ through $a$ that are fixed by $\delta$. Since $\Delta$ commutes with $\delta$ and consists of automorphisms with axis $A$, we conclude that each element of $\Delta \backslash \mathbf{1}$ fixes exactly the lines $A$ and $C_{a}$ in $\mathscr{M}_{a}$. Now $\Delta$ is an elementary abelian group acting effectively on the homotopy sphere $\mathscr{M}_{a}$ such that the set of fixed lines through $a$ forms a 0 -sphere for each non-trivial element of $\Delta$. A theorem of P. SMITH [16] yields the assertion.

Note that 8) excludes many types of finite groups (see e.g. [2] Ch. 5 , Th. 4.10; Ch. 7, Th. 6.2). In particular, the possibilities for connected Lie groups are strictly restrained.

9. Theorem. a) Commuting involutions with the same axis are equal.

b) Let $\mathscr{I}$ be a nonvoid set of commuting involutions with common center a. If there is an involution $\alpha$ with axis $A$ such that $\alpha$ commutes with each element of $\mathscr{I}$, then $\mathscr{I}$ consists of one element $\sigma$. Moreover, $\sigma=\alpha$ if $a \notin A$, and $\sigma \alpha$ is an involution with axis $C_{a}$ if $a \in A$.

Proof. Assertion a) is a corollary of 8). Let $\sigma \in \mathscr{I}$. Since $\sigma$ and $\alpha$ commute, the axis $A$ is fixed by $\sigma$. If $a \notin A$, then $A$ is an axis of $\sigma$, and $\sigma=\alpha$ by assertion a). If, on the other hand, $\alpha \neq \sigma$, then $a=A \wedge C_{a}$, and the lines $A$ and $C_{a}$ are the only lines through $a$ that are fixed by $\alpha$. Since $\sigma$ acts trivially on $\mathscr{M}_{a}$, these two lines are the only lines through $a$ that are fixed by $\sigma \alpha$, and the involution $\sigma \alpha$ is not a Baer collineation, nor has it center $a$. Assertion a) yields that the axis of $\sigma \alpha$ is $C_{a}$. Since this holds for each $\sigma \in \mathscr{I}$, we conclude that $\mathscr{I}=\{\sigma\}$, and claim b) is established.

10. Corollary. (Triangle Lemma). Let $\mathbb{M}$ be a stable plane, and let $\Phi$ and $\Delta$ be subgroups of Aut $(\mathbb{M})$ such that $\Phi$ fixes a point $x$ and $\Delta$ fixes a triangle pointwise.

a) If there are three commuting involutions in $\Phi$, then at least one of them has not an axis through $x$.

b) If there are four commuting involutions in $\Delta$, then at least one of them is a Baer collineation.

c) If there are three commuting involutions in $\Phi$ such that each of 
them has center and axis, then the centers form a non-degenerate triangle.

d) Let $\alpha, \beta, \gamma$ be three commuting involutions in $\Delta$. If none of them is a Baer collineation, then $\gamma=\alpha \beta$, and $\langle\alpha, \beta\rangle \cong \mathbb{Z} / 2 \mathbb{Z} \times \mathbb{Z} / 2 \mathbb{Z}$.

Note that the automorphism groups $\mathrm{PSL}_{3} \mathbb{H}$ and $\mathrm{E}_{6}(-26)$ of the projective planes over Hamilton's quaternions and Cayley's octonions, respectively, contain subgroups isomorphic with $(\mathbb{Z} / 2 \mathbb{Z})^{4}$ and hence 15 commuting involutions. Only three of these have axis and center, the remaining 12 are Baer collineations.

In the study of stable planes with large automorphism groups, the following will be useful:

11. Theorem. Let $\Delta$ be a locally compact group of automorphisms of a stable plane $\mathbb{M}=(M, \mathscr{M})$ with $\operatorname{dim} M=2 l$.

a) If $\Delta$ has a semi-axis, then $\operatorname{dim} \Delta \leqslant 3 l$.

b) If $\Delta$ is quasi-perspective, then $\operatorname{dim} \Delta \leqslant 3 l$.

c) If $\Delta$ is quasi-perspective and has a semi-axis, then $\operatorname{dim} \Delta \leqslant l$.

d) If $\Delta$ has a semi-axis $U$ and centralizes an involution $\sigma \in \Delta$, then $\sigma$ has axis $L_{U}, \operatorname{dim} \Delta \leqslant l$, and $\Delta$ acts freely on $\mathscr{M}_{u}\left\{L_{U}, C_{u}\right\}$ for each point $u \in U$.

Proof. i) Let $U \subseteq L_{U}$ be a semi-axis of $\Delta$. For $x \in M \backslash L_{U}$, the stabilizer $\Delta_{x}$ fixes each of the lines $x u$, where $u \in U$. The set $\{x u \mid u \in U\}$ is open in $\mathscr{M}_{x}$. Choose $u \in U$. For $y \in x u \backslash\{u\}$, the stabilizer $\Delta_{x, y}$ has two semiaxes and is therefore trivial. Assertion a) follows from the fact that $\operatorname{dim} \Delta / \Delta_{x} \leqslant \operatorname{dim} M=2 l$ and $\operatorname{dim} \Delta_{x} / \Delta_{x, y} \leqslant \operatorname{dim} x u=l$.

ii) Assume that $\Delta$ is quasi-perspective, and let $x_{1}$ be a point that is moved by $\Delta$. Choosing two points $x_{2}, x_{3}$ such that $x_{1} \notin x_{2} x_{3}$ we obtain that $\Delta_{x_{1}, x_{2}, x_{3}}$ is trivial. Since $\operatorname{dim} \Delta / \Delta_{x_{i}} \leqslant \operatorname{dim} L_{x_{i}}=l$, assertion b) follows. In case c), choose $x_{2}$ and $x_{3}$ in the semi-axis.

iii) Assume the situation of d). Obviously, the involution $\sigma$ has axis $L_{U}$, and $\Delta$ fixes $C_{u}$ for each point $u \in U$. Choosing $x_{1} \in C_{u}\{u\}$ and $x_{2}$, $x_{3} \in U$, we obtain that $\operatorname{dim} \Delta \leqslant l$ analogously to ii).

\section{References}

[1] BAER, R.: Projectivities with fixed points on every line of the plane. Bull Amer. Math. Soc. 52, 273-286 (1946).

[2] Gorenstein, D.: Finite Groups. New York: Harper \& Row. 1968. 
[3] GroH, H., Lippert, M. F., POHL, H. J.: R2-planes with 3-dimensional automorphism group fixing precisely a line. J. Geom. 21, 66-96 (1983).

[4] Hughes, D. R., PIPER, F. C.: Projective Planes. New York: Springer. 1973.

[5] KLeIN, F.: Über die sogenannte Nicht-Euklidische Geometrie. Math. Ann. 4, $573-625$ (1871).

[6] Löwen, R.: Vierdimensionale stabile Ebenen. Geom. Dedicata 5, 239-294 (1976).

[7] LöwEN, R.: Halbeinfache Automorphismengruppen von vierdimensionalen stabilen Ebenen sind quasi-einfach. Math. Ann. 236, 15-28 (1978).

[8] LöWEN, R.: Topology and dimension of stable planes: On a conjecture by $\mathrm{H}$. Freudenthal. J. Reine Angew. Math. 343, 108-122 (1983).

[9] LöwEN, R.: Stable planes admitting a classical motion group. Resultate Math. 9, $119-130$ (1986).

[10] NaGata, J.: Modern Dimension Theory. New York: Interscience. 1965.

[11] PrckerT, G.: Projektive Ebenen. Berlin: Springer. 1955.

[12] Salzmann, H.: Compact 8-dimensional projective planes with large collineation groups. Geom. Dedicata 8, 139-161 (1979).

[13] SalzmanN, H.: Automorphismengruppen 8-dimensionaler Ternärkörper. Math. Z. 166, 265-275 (1979).

[14] SalzmanN, H.: Compact 16-dimensional projective planes with large collineation groups, IV. Canad. J. Math. 39, 908-919 (1987).

[15] Sмiтh, P. A.: Fixed point theorems for periodic transformations. Amer. J. Math. 63, $1-8(1941)$.

[16] SMITH, P. A.: New results and old problems in finite transformation groups. Bull. Amer. Math. Soc. 66, 401-415 (1960).

[17] SPANIER, E. H.: Algebraic Topology. New York: McGraw-Hill. 1966.

[18] STROPPEL, M.: Stable planes. Discrete Math. To appear.

[19] STRopPEL, M.: Planar groups of automorphisms of stable planes. J. Geom. 44, $184-200$ (1992).

\section{STROPPEL}

Fachbereich Mathematik

Technische Hochschule Darmstadt

SchloBgartenstr. 7

D-6100 Darmstadt

Bundesrepublik Deutschland

\footnotetext{
Verleger: Springer-Verlag KG, Sachsenplatz 4-6, A-1201 Wien. - Herausgeber: Prof. Dr. Karl Sigmund, Institut für Mathematik der Universität, Strudlhofgasse 4, A-1090 Wien. - Redaktion: Strudlhofgasse 4, A-1090 Wien. - Hersteller: Ernst Becvar, Lichtgasse 10, A-1150 Wien. - Verlagsort: Wien. Herstellungsort: Wien. - Printed in Austria.

Offenlegung gemäß \& 25 Abs. 1 bis 3 Mediengesetz: Unternehmensgegenstand: Verlag von wissenschaftlichen Büchern und Zeitschriften. An der Springer-Verlag KG ist beteiligt: Dr. Konrad F. Springer, Sachsenplatz 4-6, A-1201 Wien, als Kommanditist zu 52,38\%. Geschäftsführer: Dr. Konrad F. Springer, Prof. Dr. Dietrich Götze, Ing. Wolfram F. Joos, Dipl.-Kfm. Claus Michaletz und Rudolf Sigle, alle Sachsenplatz 4-6, A-1201 Wien.
} 